\title{
Relationship between electrophilicity index, Hammett constant and nucleus-independent chemical shift
}

\author{
M ELANGO $^{1}$, R PARTHASARATHI $^{1}$, G KARTHIK NARAYANAN $^{2}$, \\ A MD SABEELULLAH ${ }^{2}$, U SARKAR $^{3}, \mathrm{~N} \mathrm{~S} \mathrm{VENKATASUBRAMANIYAN}^{2}$, \\ $\mathrm{V} \mathrm{SUBRAMANIAN}^{1} *$ and P K CHATTARAJ ${ }^{3} *$ \\ ${ }^{1}$ Chemical Laboratory, Central Leather Research Institute, Adyar, Chennai 600 020, India \\ ${ }^{2}$ Department of Physics, Ramakrishna Mission Vivekananda College, Mylapore, Chennai 600004 , India \\ ${ }^{3}$ Department of Chemistry, Indian Institute of Technology, Kharagpur 721 302, India \\ e-mail: ${ }^{1}$ subuchem@ @otmail.com; ${ }^{3}$ *pkc@ chem.iitkgp.ernet.in
}

MS received 21 September 2004; revised 30 December 2004

\begin{abstract}
Inter-relationships between the electrophilicity index $(\omega)$, Hammett constant $\left(o_{p}\right)$ and nucleusindependent chemical shift (NICS (1) - NICS value one å ngstrom above the ring centre) have been investigated for a series of meta- and para-substituted benzoic acids. Good linear relationships between Hammett constant vs electrophilicity and Hammett constant vs NICS (1) values have been observed. However, the variation of NICS (1) against $\omega$ shows only a low correlation coefficient.
\end{abstract}

Keywords. Electrophilicity; Hammett constant; NICS (1); reactivity; QSAR.

\section{Introduction}

Conceptual density functional theory (DFT) has been widely used to study the chemical reactivity and site selectivity of various molecular systems. ${ }^{1-3}$ The success of DFT is that it provides simple working equations to elucidate stability and reactivity. It is important to analyse the usefulness of DFT-based global reactivity descriptors in understanding the aromaticity of various hydrocarbons. ${ }^{3}$ In recent years, there has been tremendous interest in understanding the origin of the aromaticity. Schleyer et $a l^{4-8}$ have made seminal contributions in the field of aromaticity of hydrocarbons by proposing the idea of the nucleusindependent chemical shift (NICS). NICS is defined as the negative value of the absolute magnetic shielding calculated at the ring centre. NICS (0) represents the NICS value at the centre of the ring and NICS (1) is the NICS value of the molecule $1 \AA$ above the ring centre. Schleyer and his coworkers ${ }^{6}$ have established that NICS (1) provides appropriate information about the aromaticity of various hydrocarbons. Within this model, the aromatic rings are assumed to be the rings with negative values of NICS. The more the negative value of this descriptor the greater is the aromatic character of the molecular

\footnotetext{
*For correspondence
}

system. The success of NICS as a measure of the aromaticity is highly conspicous and NICS has been employed as an effective index of aromaticity. Recently, the relevance of NICS in understanding the aromaticity in benzene and related compounds has been studied. ${ }^{9}$

Development of new chemical reactivity descriptors has gained significant momentum due to their application in various areas of chemistry, biology, rational drug design and computer-aided toxicity prediction. ${ }^{10}$ In quantitative structure activity and reactivity parlance, the use of Hammett constants is well known. ${ }^{11,12}$ The Hammett equation is given by

$$
\log \left(k / k_{0}\right)=\rho \sigma_{p},
$$

where $k$ and $k_{0}$ are rate (or equilibrium) constants for the reactions of the substituted and unsubstituted compounds, $\sigma_{p}$ is the Hammett constant (substituent constant), and $\rho$ is called the reaction constant. The Hammett equation has been one of the most widely used means for the study and interpretation of organic reactions and their mechanisms.

Several attempts have been made to relate the Hammett constant with various reactivity parameters for the development of new reactivity descriptors. ${ }^{13}$ The use of electrostatic potential in the QSAR parla- 
nce has also been attempted. ${ }^{14}$ Gadre and Suresh ${ }^{15}$ have probed the relation between $\sigma_{p}$ and the most negative valued MESP $\left(V_{\min }\right)$ for several mono- and di-substituted aromatic compounds. It was found from these studies that electrostatic potential is a succinct tool in describing the reactivity of these substituted aromatic compounds. ${ }^{15}$

Formal definitions and working equations of global hardness, global softness and chemical potential are provided in several previous works. ${ }^{1-3}$ In addition to these global quantities, recently, Parr et $a l^{16}$ have proposed the global electrophilicity power (electrophilicity index, $\omega$ ) of a molecule in terms of its chemical potential and chemical hardness as,

$$
\omega=\mu^{2} / 2 \eta
$$

where $\mu \approx-(I+A) / 2$ and $\eta=(I-A) / 2$ are the electronic chemical potential and chemical hardness of the ground state of atoms and molecules respectively, approximated in terms of the vertical ionization potential $(I)$ and electron affinity $(A)$ using Koopmans' theorem. $\omega$ describes the electrophilic power of a ligand and also its propensity to soak up electrons. ${ }^{16}$ This index measures the stabilization in energy when the system acquires an additional electronic charge from the environment. By definition, it encompasses both the ability of an electrophile to acquire additional electronic charge and the resistance of the system to exchange electronic charge with the environment.

One of the important goals in physical organic chemistry is to have an appropriate description of the chemical substituents in the reactivity patterns of molecules. ${ }^{17}$ Several systematic linear relationships between substituent groups and chemical reactivities/ properties have been developed. ${ }^{18,19}$ In this context, some important results relevant to reactivity descriptors based on DFT are mentioned. Electrophilicity index has been used to classify a series of reagents present in Diels-Alder reactions and 1,3-dipolar cycloadditions. ${ }^{20}$ Good correlation between the difference in electrophilicity for diene and dienophile or dipole and dipolarophile pair and feasibility of cycloadditions has been found. ${ }^{21}$ Linear relationships between NICS and $\sigma_{p}$, and NICS and chemical hardness have been recently obtained for $p$-substituted benzyl cations. ${ }^{13 a}$ The relationship between various aromaticity indices and NICS has also been studied. ${ }^{13 \mathrm{~b}}$ Recently Domingo et $a l^{13 \mathrm{c}}$ have compared the experimental $\sigma_{p}$ values and electronic electrophilicity index $\omega$ for a series of 42 substituted ethylenes. In their work, a statistical procedure has been developed to obtain intrinsic electronic contributions to $\sigma_{p}$-based on the comparison between the experimental Hammett constant and electrophilicity index evaluated for a series of functional groups present in organic compounds. The aim of the present investigation is to extend and explore the possible inter-relationships between Hammett constant, NICS (1) and electrophilicity index for a series of meta- and para-substituted benzoic acids. Although the aromaticityhardness relationship has already been investigated, such illustrations have not been probed in detail for electrophilicity index. Since electrophilicity contains an additional term namely chemical potential, its importance has been addressed in this study to develop a more, general descriptor of chemical reactivity which has been employed to get useful insights into various problems. ${ }^{22,23}$ From the linear relationships among the various descriptors, electronic contributions to Hammett constant have been assessed.

\section{Computational details}

Figure 1 shows the structural representation of all the substituted benzoic acid molecules considered in this study. Geometries of the molecules are optimised using Gaussian $98^{24}$ employing HF method with four different basis sets. To probe the effect of basis set in the calculated values of various descriptors and the correlation coefficient, four different basis sets such as $6-311 \mathrm{G}^{*}, 6-31 \mathrm{G}^{* *}, 6-31+\mathrm{G}^{* *}$ and $6-31 \mathrm{G}^{*}$ have been chosen. The calculated vibrational frequencies for all the molecules ensured that the geometries corresponding to the minimum energy are achieved. Various reactivity descriptors have been calculated using the standard working equations described earlier. $^{1-3}$ Adopting the procedure suggested by Schleyer et al. ${ }^{4}$ NICS (1) values have been calcula-

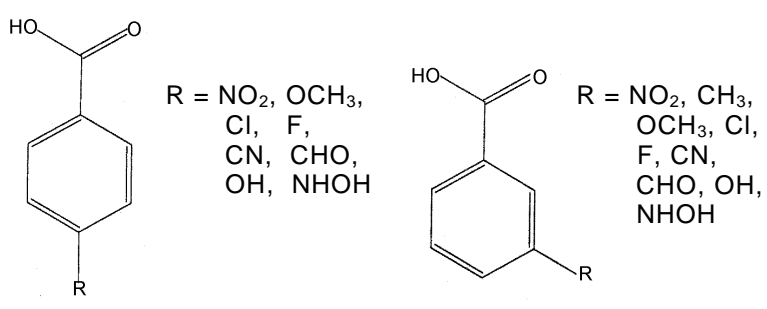

Figure 1. Structures of eight para-substituted benzoic acids and nine meta-substituted benzoic acids. 
ted. Linear regression analysis has been performed to obtain the relationship between Hammett constant and NICS (1) with $\omega$. The $\sigma_{p}$ values for the various molecular systems have been taken from the earlier reports. $^{11}$

\section{Results and discussion}

Calculated $R^{2}$ values for various interrelations between the $\sigma_{p}$, NICS (1), and $\omega$ are presented in table 1. Figure 2 a represents the calculated Hammett constants $\left(\sigma_{p}{ }^{\text {fit1 }}\right)$ vs experimental $\sigma_{p}$. The $\sigma_{p}{ }^{\text {fit1 }}$ are obtained from the following linear regression equation,

$$
\sigma_{p}{ }^{\text {fitl }}=0 \cdot 027^{*} \omega+0 \cdot 865
$$

It is evident from the $R^{2}$ values presented in table 1 and the plot represented in Figure $2 \mathrm{a}$ that there is fairly good linear relation between the Hammett constants and the electrophilicity index. The reason for good correlation between $\sigma_{p}$ and $\omega$ can be attributed to the fact that $\omega$ is a measure of the electrophilic power of the group and the sign of $\sigma_{p}$ describes electron-withdrawing or -donating nature of the substituent group. A positive value of $\sigma_{p}$ ensures that the substituent group is electron-withdrawing in nature and the reactivity of the molecule is expected to be high, on the other hand a negative value implies the electron-donating nature of the substituent and the reactivity of the molecule is thus less. In a recent study, Domingo et $a l^{13 \mathrm{c}}$ have observed a logarithmic relation between $\sigma_{p}$ and $\omega$. However, for the substituent groups considered in this study, we have found a linear correlation between the Hammett constants and electrophilicity index. The close agreement between $\sigma_{p}{ }^{\text {fit1 }}$ and $\sigma_{p}$ ensures good correlation between these descriptors of chemical reactivity and stability. The calculations have also been made using different basis sets to obtain the best estimates of various parameters. There is no unique basis set that provides better estimates of correlation coefficient for various interrelationships. In this case, the 6-311G* basis set yields better correlation between $\sigma_{p}$ and $\omega$ values.

NICS (1) ${ }^{\text {fit }}$ represents the NICS (1) values obtained from linear regression analysis between NICS (1) values calculated using Schleyer's approach and $\omega .{ }^{4}$ The variation of NICS(1) fit vs NICS (1) is depicted in figure $2 \mathrm{~b}$. Although there is a linear relationship between NICS (1) and electrophilicity index, the $R^{2}$ value for this fit is $0 \cdot 51$. It is an established fact that individually NICS (1) and $\omega$ describe the aromaticity and chemical reactivity respectively. Hence it is natural to expect a better correlation bet-

(a)

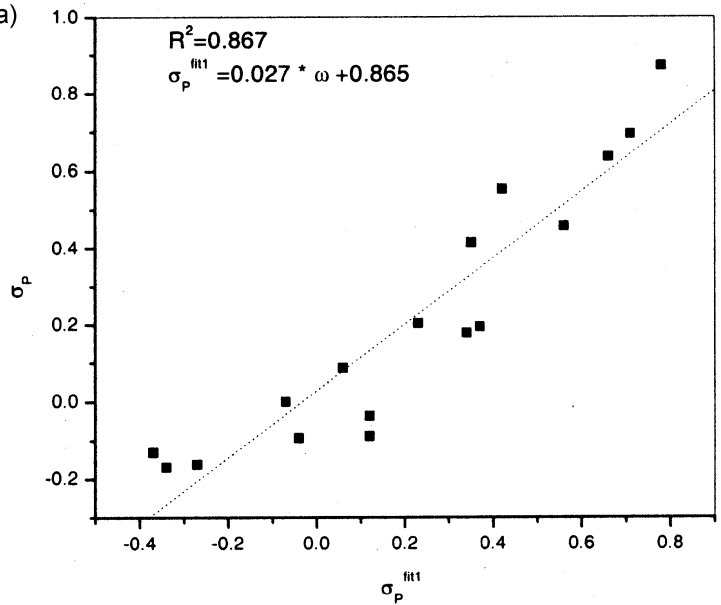

(b)

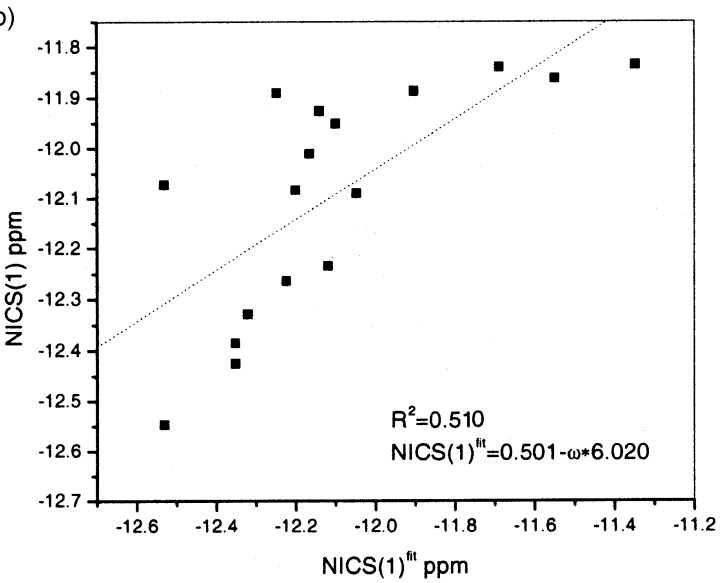

(c)

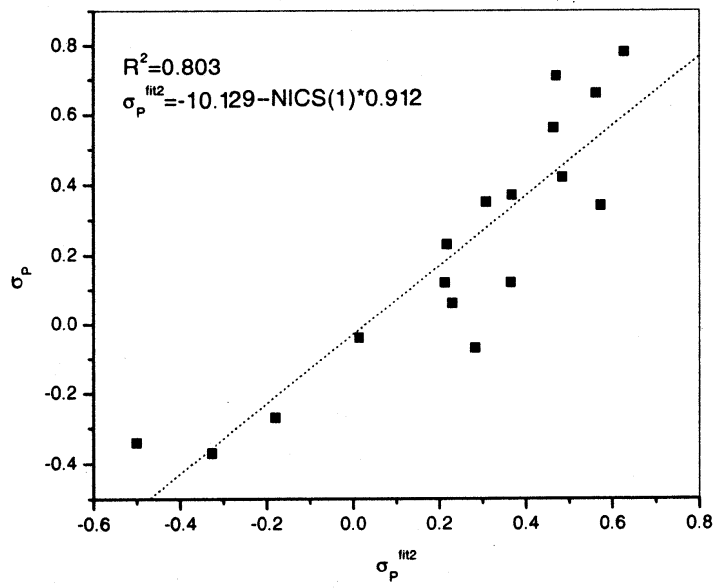

Figure 2. Linear plots between (a) $\sigma_{p}$ vs calculated $\sigma_{p}{ }^{\text {fit } 1}$ at HF/6-311G* level, (b) NICS (1) vs NICS (1) $)^{\text {fit }}$ at HF/ 6-311G* level, and (c) $\sigma_{p}$ vs $\sigma_{p}{ }^{\text {fit2 }}$ at $\mathrm{HF} / 6-31+\mathrm{G}^{* *}$ level. 
Table 1. $R^{2}$ values for various plots between $\sigma_{p}$, NICS (1), and $\omega$ at HF level with four different basis sets.

\begin{tabular}{llll}
\hline & $\sigma_{p}$ & $\omega$ & NICS (1) \\
\hline$\sigma_{p}$ & $1.000^{*}$ & & \\
& $1.000^{\circ}$ & & \\
& $1.000^{+}$ & & \\
& $1.000^{\circ}$ & & \\
$\omega$ & $0.867^{*}$ & $1.000^{*}$ & \\
& $0.857^{\circ}$ & $1.000^{\circ}$ & \\
& $0.854^{+}$ & $1.000^{+}$ & \\
& $0.753^{\circ}$ & $1.000^{\circ}$ & \\
NICS (1) & $0.740^{*}$ & $0.473^{*}$ & $1.000^{*}$ \\
& $0.721^{\circ}$ & $0.445^{\circ}$ & $1.000^{\circ}$ \\
& $0.753^{+}$ & $0.508^{+}$ & $1.000^{+}$ \\
& $0.803^{\circ}$ & $0.438^{\bullet}$ & $1.000^{\circ}$ \\
\hline$* H F / 6-311 \mathrm{G}^{*}{ }^{\circ} \mathrm{HF} / 6-31 \mathrm{G}^{* *},{ }^{+} \mathrm{HF} / 6-31 \mathrm{C}^{*},{ }^{\circ} \mathrm{HF} / 6-31+\mathrm{G}^{* *}$
\end{tabular}

*HF/6-311G*, ${ }^{*} \mathrm{HF} / 6-31 \mathrm{G}^{* *}$; ${ }^{+} \mathrm{HF} / 6-31 \mathrm{G}^{*}$; ${ }^{\bullet} \mathrm{HF} / 6-31+\mathrm{G}^{* *}$

ween these two quantities. Contrary to this expectation, regression analysis reveals that there is no good correlation between aromatic criteria and $\omega$. However, the inverse relations between NICS (1) values for all molecules and $\omega$ indicate that highly aromatic systems have low reactivity as exemplified by the $\omega$ values. Since $\omega$ contains a hardness term in the denominator which is a descriptor of stability, it is expected that aromaticity and $\omega$ will exhibit an inverse linear relationship. However, the electrophilicity encompasses a chemical potential term in addition to hardness. It is possible to note from the $R^{2}$ value obtained from the linear least square analysis that chemical potential has profound influence on the relationship. It is important to point out here that the dimensions of chemical hardness and electronegativity are the same and that the square of the chemical potential is the same as the square of the electronegativity. It is expected that electrophilicity and electronegativity will show the similar trends in most cases which is in conformity with chemical intuition.

The plot of $\sigma_{p}^{\text {fit2 }}$ vs $\sigma_{p}$ is depicted in figure 2c. From the regression analysis between $\sigma_{p}$ and NICS (1) values, $\sigma_{p}{ }^{\text {fit2 }}$ has been calculated. It is evident from the results that there is a good correlation between $\sigma_{p}$ and NICS (1) values.

\section{Conclusion}

It is possible to obtain linear relationships between Hammett constants of various substituted benzoic acids with their corresponding electrophilicity indices. Evidently, electrophilicity index can be used in
QSAR parlance. In this context, it is pertinent to mention that electrophilicity contains information about both electron transfer (chemical potential) and stability (hardness), it is expected to be a better descriptor of global chemical reactivity, as has been authenticated in many studies. ${ }^{13 c, 22,23}$ Similarly the linear regression analysis of the data between NICS (1) and $o_{p}$ showed good correlation confirming the high aromaticity-low reactivity pattern. When compared to these linear variations, the correlation coefficient of NICS (1) vs $\omega$ is found to be lower. This may be attributed to the role of the chemical potential term present in the definition of electrophilicity index. Results from the linear regression analysis and $\sigma_{p}{ }^{\text {fit1 }}$ and $\sigma_{p}{ }^{\text {fit2 }}$ values indicate that it is highly feasible to obtain information about electronic contributions to the Hammett constants which may serve as an effective tool to forecast chemical reactivity.

\section{Acknowledgements}

We thank the Council of Scientific and Industrial Research, New Delhi for financial assistance and $\mathrm{Mr}$ D R Roy for help in manuscript preparation.

\section{References}

1. Parr R G and Yang W 1989 Density functional theory of atoms and molecules (New York: Oxford University Press)

2. Geerlings P, De Proft F and Langenaeker W 2003 Chem. Rev. 1031793

3. De Proft F and Geerlings P 2001 Chem. Rev. 101 1451

4. Schleyer P v R, Maercker C, Dransfield A, Jiao H and Hommes N J R v E 1996 J. Am. Chem. Soc. 118 6317

5. Jiao H and Schleyer P v R 1996 Angew. Chem., Int. Ed. Engl. 352383

6. Schleyer P v R, Jiao H, Hommes N J R v E, Malkin V G and Malkina O L 1997 J. Am. Chem. Soc. 119 12669

7. (a) Schleyer P v R, Manoharan M, Wang Z X, Kiran B, Jiao H, Puchta R and Hommes N J R v E 2001 Org. Lett. 3 2465; (b) Schleyer P v R, Kiran B, Simion D V and Sorensen T S $2000 \mathrm{~J}$. Am. Chem. Soc. 122 510; (c) Castro C, Isborn C M, Karney W L, Mauksch M and Schleyer P v R 2002 Org. Lett. 4 3431; (d) Cyranski M K, Krygowski T M, Katritzky A R and Schleyer P v R 2002 J. Org. Chem. 67 1333

8. (a) Havenith $\mathrm{R} \mathrm{W}$ A, Jiao H, Jenneskens L W, van Lenthe J H, Sarobe M, Schleyer P v R, Kataoka M, Necula A and Scott L T 2002 J. Am. Chem. Soc. 124 
2363; (b) Jung Y, Heine T, Schleyer P v R and HeadGordon M 2004 J. Am. Chem. Soc. 1263132

9. (a) Saieswari A, Priyakumar U D and Sastry G N 2003 J. Mol. Struct. (Theochem). 663 145; (b) Priyakumar U D and Sastry G N 2003 Proc. Indian Acad. Sci. (Chem. Sci.) 115 49; (c) Priyakumar U D and Sastry G N 2003 J. Org. Chem. 67 271; (d) Priyakumar U D and Sastry G N 2000 J. Am. Chem. Soc. 122 11173

10. Chattaraj P K, Nath S and Maiti B 2003 Reactivity descriptors. In Computational medicinal chemistry for drug discovery (eds) J Tollenaere, $\mathrm{P}$ Bultinck, $\mathrm{H}$ D Winter and W Langenaeker (New York: Marcel Dekker) ch. 11, pp 295-322

11. Hansch C, Leo A and Taft R W 1991 Chem. Rev. 91 165

12. March J 1998 Advanced organic chemistry: Reactions, mechanisms and structure (New York: Wiley \& Sons)

13. (a) Krygowski T M and Cyranski M 1996 Tetrahedron 52 1713; (b) Morao I and Hillier I H 2001 Tetrahedron Lett. 42 4429; (c) Domingo L R, Perez P and Contreras R 2003 J. Org. Chem. 686060

14. (a) Gadre S R and Suresh C H 1997 J. Org. Chem. 62 2625; (b) Gadre S R and Shirsat R N 2000 Electrostatics of atoms and molecules: An educational monograph (Hyderabad: Universities Press)

15. Gadre S R and Suresh C H 1998 J. Am. Chem. Soc. 1207049
16. Parr R G, Szentpaly L V and Liu S 1999 J. Am. Chem. Soc. 1211922

17. Exner O 1972 In Advances in linear free energy relationships (eds) N B Chapman and J Shorter (London: Plenum)

18. (a) Franke R 1984 Theoretical drug design methods (Amsterdam: Elseveir) p 115; (b) Gupta S P, Singh P and Bindal M C 1983 Chem. Rev. 83 633; (c) Gupta S P 1991 Chem. Rev. 91 1109; (d) Hansch C H, Hoekman D, Leo A, Weininger D and Selassie D D 2002 Chem. Rev. 102783

19. Li Y and Evans N S 1995 J. Am. Chem. Soc. 117 7756; Perez P, Simon-Manso Y, Aizman A, Fuentealba P and Contreras R $2000 \mathrm{~J}$. Am. Chem. Soc. 122 4756; Thanikaivelan P, Subramanian V, Raghava Rao J and Nair B U 2000 Chem. Phys. Lett. 323 59; Chang C M 2003 J. Phys. Chem. B107 5190

20. Perez P, Domingo L R, Aurell M J and Contreras R 2003 Tetrahedron 593117

21. Domingo L R, Arno M, Contreras R and Perez $\mathrm{P}$ 2002 J. Phys. Chem. A106 952

22. Parthasarathi R, Subramanian V, Roy D R and Chattaraj P K 2004 Bioorg. Med. Chem. 12, 5533

23. Parthasarathi R, Padmanabhan J, Subramanian V, Maiti B and Chattaraj P K 2003 J. Phys. Chem. A107 10346

24. GAUSSIAN 981998 Revision A.7, Gaussian Inc., Pittsburgh, PA 